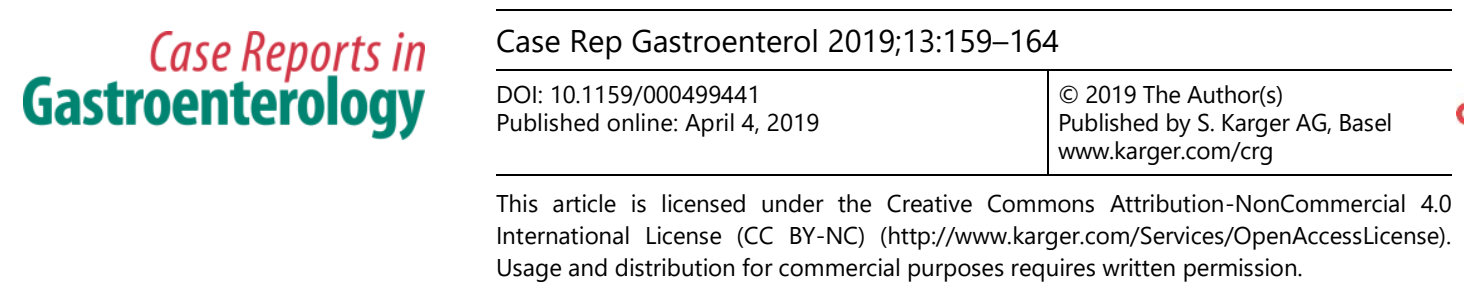

\title{
Laparoscopic Resection of a Primary Retroperitoneal Mucinous Cystadenoma
}

\author{
Ryota Koyama Yoshiaki Maeda Nozomi Minagawa Toshiki Shinohara \\ Tomonori Hamada \\ Department of Gastrointestinal Surgery, Hokkaido Cancer Center, Sapporo, Japan
}

\section{Keywords}

Primary retroperitoneal mucinous cystadenoma - Retroperitoneal tumor - Laparoscopic surgery

\begin{abstract}
Primary retroperitoneal mucinous cystadenoma (PRMC) is a rare cystic lesion occurring mostly in women with a histological analogy to ovarian mucinous cystadenoma. The tumor is difficult to detect during early stages because it causes symptoms only when it grows large enough to be palpable or to displace the adjacent internal organs. The primary treatment is resection, but the optimal surgical approach remains poorly known. We report the case of a 41-year-old woman who complained of right-sided intermittent abdominal pain. Imaging studies revealed a right retroperitoneal smooth cystic lesion $(50 \mathrm{~mm})$ without invasive features. Laparoscopic resection was then performed. During surgery, a right retroperitoneal mass with no connection to neighboring tissues was found. The tumor, wrapped by retroperitoneal fat tissue, was resected and removed from the body without exposure. Furthermore, histopathological findings indicated PRMC. The patient was discharged without any complications and observed to have no recurrence 6 months postoperatively.




\section{Case Reports in Gastroenterology}

Case Rep Gastroenterol 2019;13:159-164

DOI: 10.1159/000499441

(c) 2019 T www.karger.com/crg

Koyama et al.: Laparoscopic Resection of a Primary Retroperitoneal Mucinous Cystadenoma

\section{Introduction}

Primary retroperitoneal mucinous cystadenoma (PRMC) is a rare cystic lesion occurring in the retroperitoneum. The histogenesis remains unclear because no epithelial tissue originally exists in the retroperitoneum. The diagnosis is determined by pathological findings instead of radiological findings; therefore, surgical resection is required for both diagnosis and treatment. Laparoscopic resection of PRMC has increasingly been applied even if radiological signs, such as enhanced mural nodule or calcification, indicate malignancy [1]. However, the criteria for laparoscopic resection of retroperitoneal lesions remain unknown. Herein, we report a case of the successful laparoscopic resection of PRMC and review the literature.

\section{Case Presentation}

Our patient was a 41-year-old female without any previous medical history. She complained of right-sided intermittent abdominal pain that started several months before her first visit to our hospital. Her abdomen was soft and flat without any tenderness or palpable mass. Laboratory examination showed a slightly elevated C-reactive protein level $(0.21 \mathrm{mg} / \mathrm{dL})$, but complete blood count and serum chemistry were all within normal limits. Enhanced abdominal computed tomography scan revealed a right retroperitoneal well-circumscribed unilocular cystic mass of $50 \times 22 \times 30 \mathrm{~mm}$ in size with no signs of invasion to the surrounding tissue (Fig. 1a). Mural nodules and a major feeding artery were not found. Magnetic resonance imaging showed homogeneous low intensity on T1-weighted imaging and high intensity on T2-weighted imaging (Fig. 1b). Abdominal ultrasound revealed a smooth cystic lesion with distinct borders that showed partial calcification of the cystic wall. Esophagogastroduodenoscopy and colonoscopy were normal, and serum tumor markers (carcinoembryonic antigen [CEA] and cancer antigen 19-9 [CA19-9]) were within normal limits.

The patient agreed to undergo surgical resection despite the possibility that the rightsided abdominal pain might be unrelated to the right retroperitoneal mass. The patient was placed in a spine position. A pneumoperitoneum was created using carbon dioxide at $8 \mathrm{~mm}$ Hg. A 12-mm-long trocar was inserted through the umbilicus for laparoscopy. In addition, 12$\mathrm{mm}$ - and 5-mm-long trocars were placed in the left upper and lower quadrants, respectively, for the operating trocar. For the assistant surgeon, 5-mm-long trocars were placed in the right upper and lower quadrants separately. Appendectomy was also performed because the appendix was slightly inflamed, and a causal relationship between the complaint and inflamed appendix could not be ruled out. The patient was tilted to lower the head to keep the transverse colon away from the surgical field. Furthermore, a lateral approach was performed to mobilize the right-sided colon, thereby leading to a visualization of the tumor contour (Fig. 2a). Using laparoscopic coagulation shears, we resected the retroperitoneum following the surgical margin at approximately $2 \mathrm{~cm}$ from the cystic wall, which was surrounded with retroperitoneal fat tissue (Fig. $2 \mathrm{~b}$ ). Since the tumor had direct contact with the iliopsoas muscle without invasion, the dissecting layer was set along the iliopsoas muscle on the dorsal side (Fig. 2c). Nerves and ureter were preserved. The isolated tumor was extracted from the elongated umbilical incision without spillage of the content and then placed in an extraction bag. Operation time was $101 \mathrm{~min}$, and blood loss was $11 \mathrm{~mL}$.

The pathological diagnosis was a benign retroperitoneal cyst with no evidence of malignancy. The size was $55 \mathrm{~mm}$ in maximum with surrounding fat tissue (Fig. 3a, b), and the cystic lesion contained relatively clear serous fluid with elevated tumor marker levels (CEA, 4,665 


\section{Case Reports in Gastroenterology}

Case Rep Gastroenterol 2019;13:159-164

DOI: $10.1159 / 000499441$

(c) 2019 The Author(s). Published by S. Karger AG, Basel www.karger.com/crg

Koyama et al.: Laparoscopic Resection of a Primary Retroperitoneal Mucinous Cystadenoma

$\mathrm{ng} / \mathrm{mL}$ and cancer antigen 125 [CA125], 21,040 U/mL); however, the CA19-9 level was normal (Fig. 3c). Histopathological findings showed that the cystic wall consisted of thin fibrous tissues with partial single-layered cuboidal-to-flat glandular epithelium without cellular atypia (Fig. 4a, b). Immunohistochemistry revealed keratin AE1/AE3 positivity and CDX2 negativity (Fig. 4c, d). Therefore, the final diagnosis of PRMC was made. Further, the simultaneously resected appendix was normal. The patient was discharged on postoperative day 9 without any complications.

\section{Discussion}

Typically, $70-80 \%$ of all primary retroperitoneal neoplasms are potentially malignant [2]. Radiographical findings can narrow the differential diagnosis, but the final diagnosis largely depends on the pathological findings [3]. Therefore, surgical resection is required for the diagnosis, and the laparoscopic approach for retroperitoneal lesions is increasingly applied. PRMC is a rare benign cystic lesion. In retroperitoneal mucinous adenocarcinoma, male sex and solid mural nodules in the cyst are significant predictive factors of malignancy [1]. The intriguing question is whether or not a primary retroperitoneal cystadenoma progresses into a malignant lesion. However, the answer remains elusive with no previous case reports indicating this progressive model.

Four hypotheses have been suggested for the histogenesis of primary retroperitoneal cystadenoma [4]. First, such tumors originate from the seeding of ectopic ovarian tissue in the retroperitoneum. This hypothesis is supported by the histological analogy to mucinous cystadenoma in the ovary and the fact that nearly all PRMC cases are women. Second, PRMCs may arise from teratoma wherein the mucinous epithelial cells have overgrown all other components of the teratoma tissue. Third, PRMCs may originate from an enterogenic duplication cyst. Finally, PRMC may arise from the invagination of the peritoneal mesothelium that is entrapped and undergoes mucinous metaplasia and cyst formation. This last observation is supported by the immunohistochemical staining of the biopsy, where estrogen and progesterone receptors are mostly negative. However, the true cause remains unknown.

Although our case is consistent with PRMC, where some monolayered epithelial linings remain along the fibrous wall, similarities with a simple fibrous pseudocyst were still observed [5]. Nonetheless, we ruled out a simple fibrous cyst on two grounds. First, epithelial cell lining existed on the capsule, although most of them were detached from the specimen. Second, the CEA and CA125 levels in the cystic lesion were significantly elevated, indicating a neoplastic lesion.

In terms of surgical approach, open surgery is still a safe and effective way and is especially chosen in cases of malignancy, large tumor size, and invasion to other organs. However, laparoscopic resection of a retroperitoneal lesion is increasingly reported. The transabdominal laparoscopic approach is the most frequent, and the retroperitoneal laparoscopic approach is also reported [6]. The transabdominal approach enables surgeons to operate in a large working space and to look for other intra-abdominal abnormalities simultaneously, but it requires mobilization of the bowels to expose the retroperitoneum, putting the bowels at risk of damage. Conversely, the retroperitoneal approach has a smaller working space, and the surgical field is limited to the retroperitoneal space. If the contents of the cystic lesion spills over due to accidental perforation of the cystic wall, the retroperitoneal approach has a smaller risk of abdominal dissemination than the transabdominal approach. The most challenging problem of the retroperitoneal approach is having a small working space. Fujita et al. 
Koyama et al.: Laparoscopic Resection of a Primary Retroperitoneal Mucinous Cystadenoma

[6] used a SAND balloon catheter, which is commonly used for the resection of ovarian cysts, to extract the cystic content via the trocar site.

In conclusion, when a retroperitoneal cystic lesion is detected, the feasibility of a laparoscopic resection should be pursued when no apparent contraindication exists.

\section{Statement of Ethics}

The authors have no ethical conflicts to disclose.

\section{Disclosure Statement}

The authors have no conflicts of interest to declare.

\section{References}

1 Lee SE, Oh HC, Park YG, Choi YS, Kim MK. Laparoscopic excision of primary retroperitoneal mucinous cystadenoma and malignant predicting factors derived from literature review. Int J Surg Case Rep. 2015;9:130-3.

2 Neville A, Herts BR. CT characteristics of primary retroperitoneal neoplasms. Crit Rev Computed Tomogr. 2004;45(4):247-70.

3 Subramony C, Habibpour S, Hashimoto LA. Retroperitoneal mucinous cystadenoma. Arch Pathol Lab Med. 2001 May;125(5):691-4.

4 Blount SE, Cobb C, Raza AS. Non-pancreatic retroperitoneal mucinous neoplasms and a discussion of the differential diagnosis. J Gastrointest Oncol. 2016 Apr;7 Suppl 1:S75-80.

5 Ahn J, Chandrasegaram MD, Alsaleh K, Woodham BL, Teo A, Das A, et al. Large retroperitoneal isolated fibrous cyst in absence of preceding trauma or acute pancreatitis. BMC Surg. 2015 Mar;15(1):25.

6 Fujita K, Yamamoto Y, Yamaguchi S. Laparoscopic resection of primary retroperitoneal mucinous cystadenoma by retroperitoneal approach. Int J Urol. 2011 Aug;18(8):607-8.
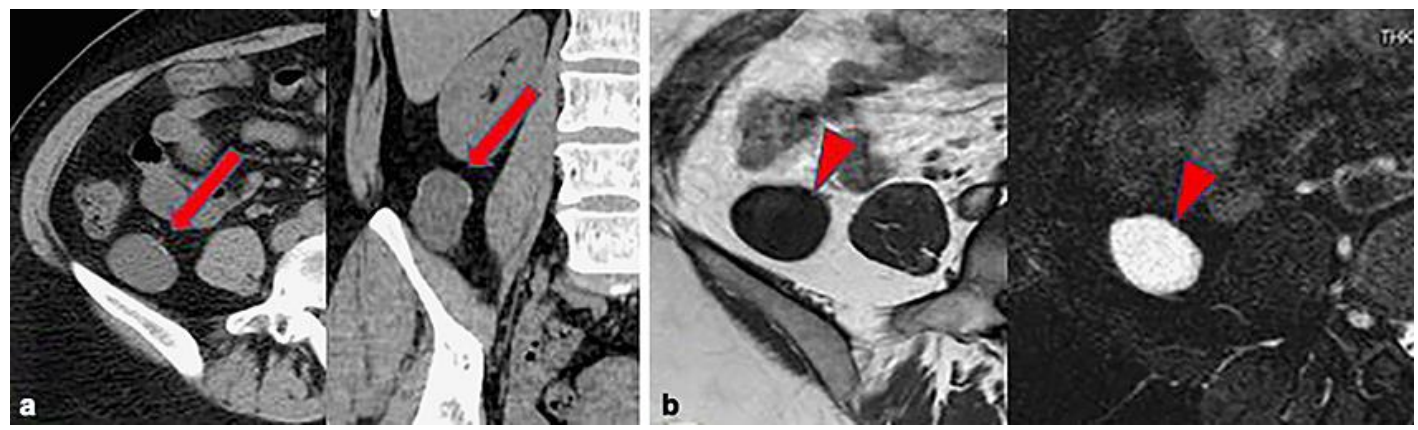

Fig. 1. Computed tomography (CT) and magnetic resonance imaging (MRI). a CT shows a $50 \times 22 \times 30 \mathrm{~mm}$, right, retroperitoneal, well-circumscribed, unilocular, cystic mass with calcification (arrow). b The intensity of the content of the cystic lesion was low on T1-weighted image and high on T2-weighted image (arrowhead). 


\section{Case Reports in Gastroenterology}

\begin{tabular}{l|l}
\hline Case Rep Gastroenterol 2019;13:159-164 \\
\hline DOI: 10.1159/000499441 & $\begin{array}{l}\text { ○ 2019 The Author(s). Published by S. Karger AG, Basel } \\
\text { www.karger.com/crg }\end{array}$ \\
\hline
\end{tabular}

Koyama et al.: Laparoscopic Resection of a Primary Retroperitoneal Mucinous Cystadenoma
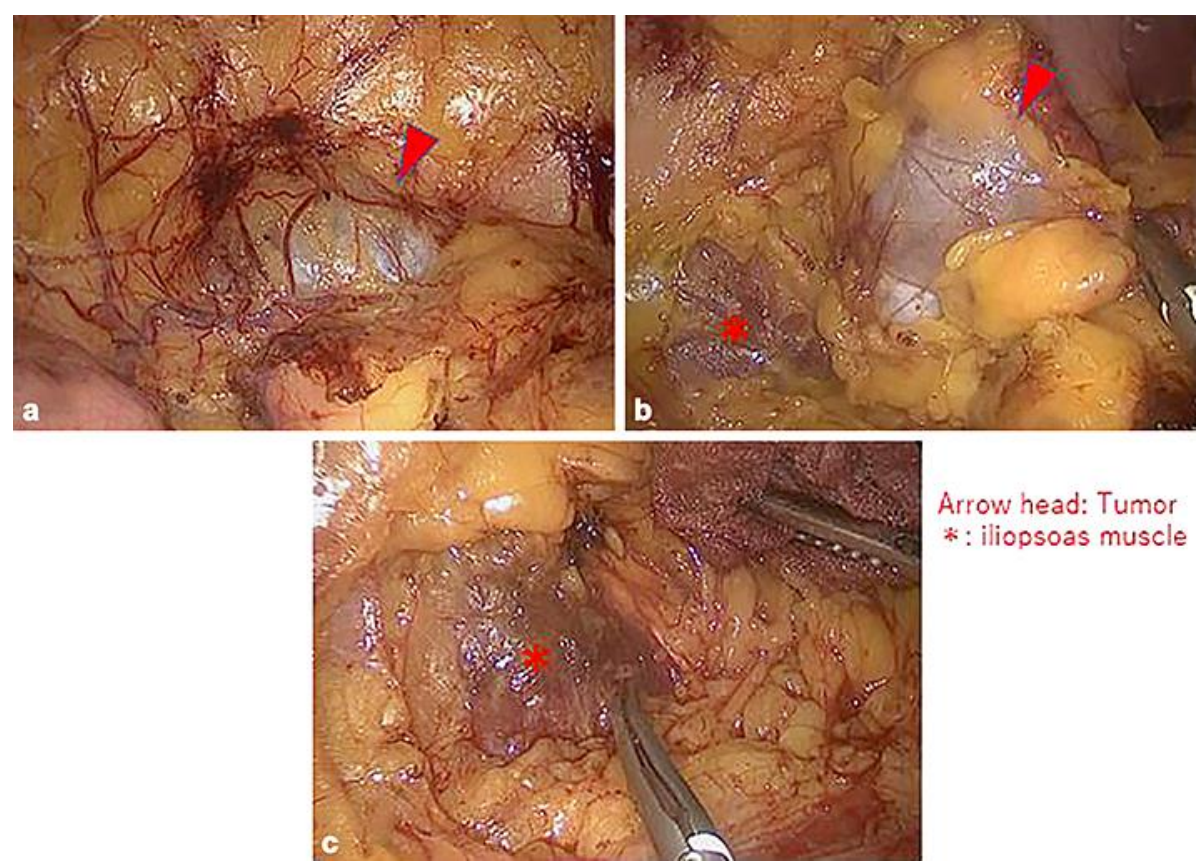

Arrow head: Tumor

*: iliopsoas muscle

Fig. 2. Intraoperative finding. a The tumor was a grayish mass with slight protrusion to the peritoneum. b The retroperitoneal fat tissue surrounding the tumor was attached as the surgical margin. $\mathbf{c}$ On the dorsal side, the iliopsoas muscle (indicated by a *) was exposed.
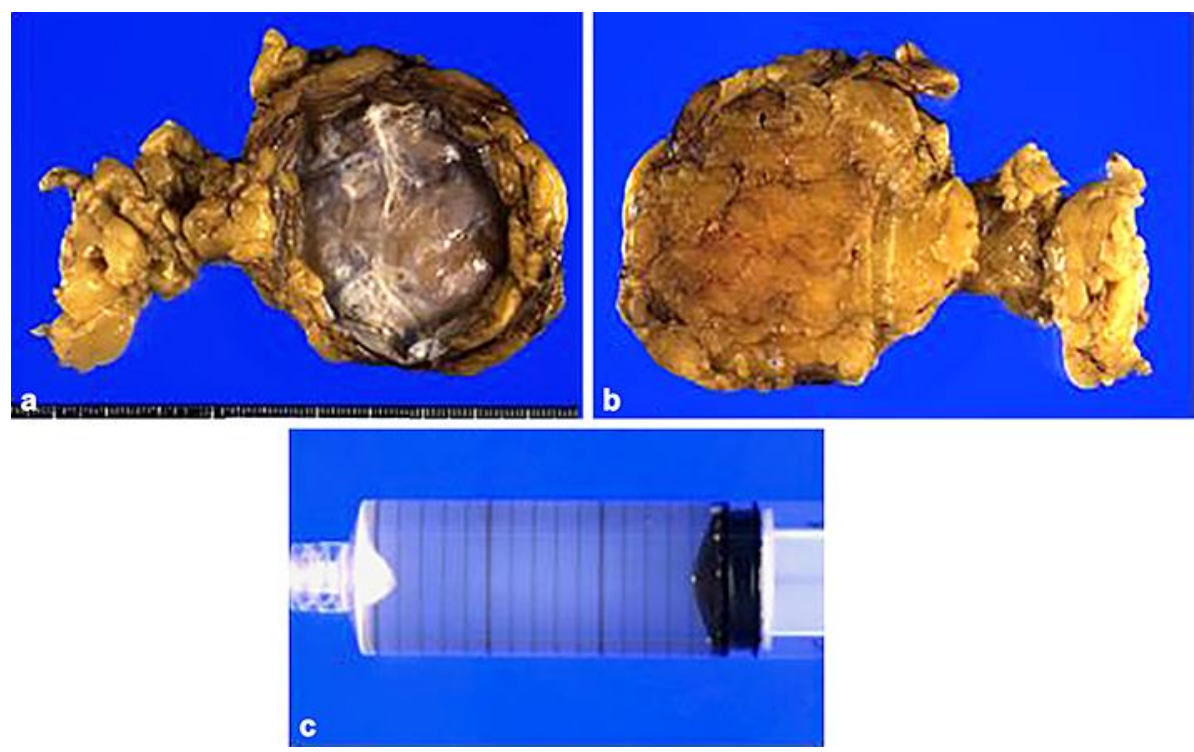

Fig. 3. Macroscopic findings of the resected specimen. a The resected specimen was monolocular and mostly a fibrous cystic lesion with calcification of the cystic wall but without mural nodules. $\mathbf{b}$ Dorsal view of the specimen. $\mathbf{c}$ The content inside the lesion was a slightly mucinous yellowish fluid. 


\section{Case Reports in \\ Gastroenterology}

\begin{tabular}{l|l}
\hline Case Rep Gastroenterol 2019;13:159-164 \\
\hline DOI: 10.1159/000499441 & $\begin{array}{l}\text { (C) 2019 The Author(s). Published by S. Karger AG, Basel } \\
\text { www.karger.com/crg }\end{array}$ \\
\hline
\end{tabular}

Koyama et al.: Laparoscopic Resection of a Primary Retroperitoneal Mucinous Cystadenoma
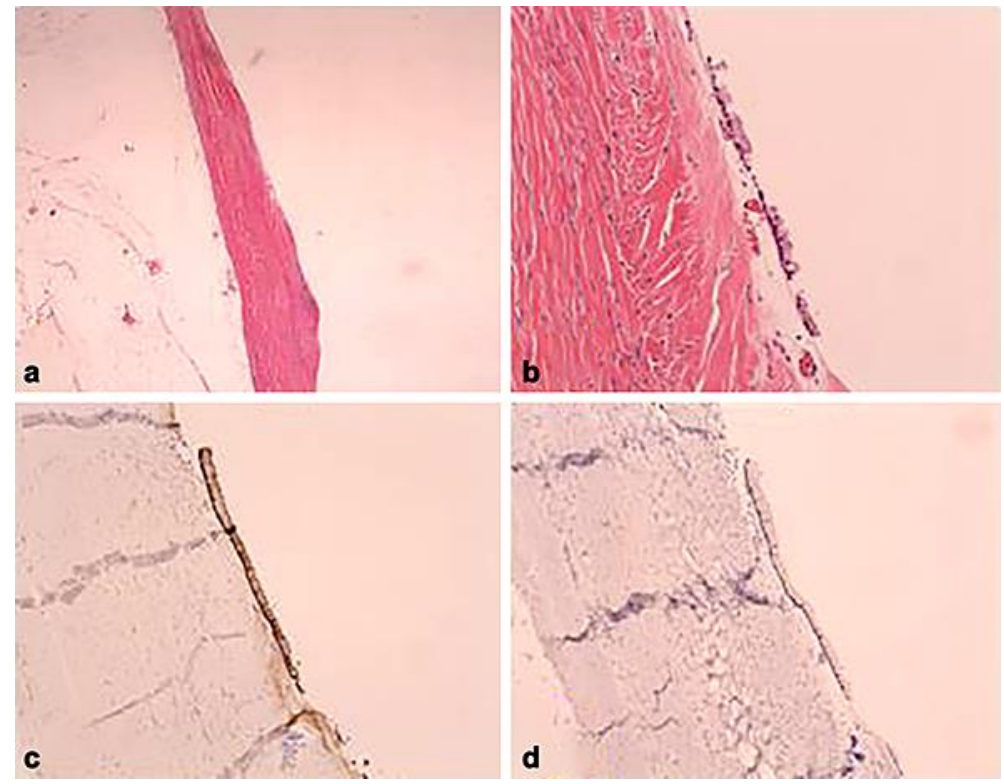

Fig. 4. Histopathology of the resected specimen. $\mathbf{a}$, $\mathbf{b}$ The cystic wall consisted of thin fibrous tissues with a partial single-layered cuboidal-to-flat glandular epithelium without cellular atypia (a: HE. $\times 20$. b: $\times 100$ ) Immunohistochemical staining was positive for keratin AE1/AE3 (c) and negative for CDX2 (d). 